\title{
THz Quantum Cascade Lasers with low effective mass active region
}

\author{
M. Brandstetter, M. Krall, M. Kainz, S. Schönhuber, C. Deutsch, T. Zederbauer, A.M. Andrews, G. Strasser, K. \\ Unterrainer (invited talk) \\ Photonics Institute and Center for Micro\&Nanostructures \\ Technische Universität Wien \\ Vienna, Austria
}

\begin{abstract}
The development of InGaAs based terahertz quantum cascade lasers is reported. This material system is an attractive alternative to the used GaAs/AlGaAs due the smaller effective mass. Using a symmetric quantum cascade design allows to understand the barrier interface and to realize high performance devices.
\end{abstract}

Keywords-THz, quantum cascade laser; intersubband, bandstructure engineering

Terahertz quantum cascade lasers (THz QCLs) are promising candidates as compact and efficient sources in realworld applications. There was a rapid development towards higher operating temperatures and optical output power. However, the maximum operating temperature is still around $200 \mathrm{~K}$ which hampers a widespread use in applications. Thus new approaches are required. The benefit of an additional confinement in the lateral direction was already demonstrated with high magnetic fields or in quantum dots a couple of years ago. However, a practical implementation of such a confinement is still a technological challenge [1]. The second, more conventional approach is to explore alternative material systems. In theory, QCLs could be realized in a variety of material systems, a consequence of band structure engineering. Unlike in diode lasers, the optical transition energy is not determined by the band gap, which is an intrinsic material parameter. The optical transition is tailored by adjusting the widths of quantum wells and barriers in a semiconductor heterostructure. Thus QCL operation has been demonstrated over an incredibly broad spectral range reaching from 3-300 $\mu \mathrm{m}$. At first glance, any semiconductor heterostructure is suitable for a QCL, but there are a lot of technological restrictions. QCL growth is demanding: almost monolayer accuracy, atomically sharp interfaces, lattice-matching, etc. On the other hand, material parameters also heavily affect QCL performance. As a result, the InGaAs/InAlAs/InP (lattice matched and strained), is the choice when it comes to the fabrication of high performance QCL in the mid-infrared region. For mid-infrared QCLs GaAs/AlGaAs devices, which can be grown with extremely high quality, are not competitive because of a lower conduction band offset, a higher effective electron mass and disadvantages for waveguide fabrication.
$\mathrm{THz}$ QCLs, on the other hand, are preferentially realized in the GaAs/AlGaAs material system using rather low aluminum contents in the range of $10-15 \% .1$ The biggest difference to their mid-infrared ancestors is the optical transition energy that is one order of magnitude smaller $(\approx 10 \mathrm{meV})$ and below the reststrahlenband. Therefore, a high conduction band offset is not required. Precise injection into the upper laser state and depopulation of the lower laser state for population inversion becomes crucial though. Employing low conduction band offsets avoids extremely thin barriers in the active region. Hence, the structures are less sensitive to growth imperfections compared to similar structures with a higher conduction band offset. InGaAs has a one third lower effective electron mass, allowing higher optical gain, but so far, InGaAs/InAlAs THz QCLs have not reached the operating temperatures of GaAs/AlGaAs devices [2].

Interface roughness, charged impurity and alloy scattering have dramatic effects on the device performance and should be minimized. This non-radiative scattering in turn is strongly related to the growth quality (interfaces, background doping levels, etc.).

We have pioneered InGaAs/GaAsSb lattice-matched to InP as a novel material system for intersubband devices [3]. The first Al-free mid-infrared QCL and QWIP devices have been demonstrated just a few years ago. The material system is similar to the standard InP-based InGaAs/InAlAs but with a different barrier. The replacement of the barrier material reduces the conduction band offset to $360 \mathrm{meV}$ and makes it actually less attractive for mid-infrared devices. However, it makes it more attractive for the fabrication of THz QCLs because it allows the design of thicker barriers compared to InAlAs [5]. The effective electron mass of the barrier is also much lower, which further enhances the coupling between wells.

Besides favorable properties for $\mathrm{THz}$ QCLs, the GaAsSb material exhibits some drawbacks. Doping of GaAsSb is not well studied. The much bigger issue is interface asymmetry. Usually the inverted interface (switching from the barrier to the well material) is rougher compared to the normal interface. Therefore, interface asymmetry is closely related to the growth direction. THz QCLs are very sensitive to elastic scattering and interface asymmetry/growth direction has to be taken into account. 
The active region designs are based on the three-well phonon depletion scheme [4]. Due to interface asymmetry, the samples were grown with a certain growth order. When biased, the electron transport occurs in growth direction and against the normal, sharper interfaces. Subsequently, the samples were processed in double-metal waveguides.

InGaAs/GaAsSb is a genuinely attractive material system for $\mathrm{THz}$ QCLs [7]. The replacement of the standard InAlAs barrier material with GaAsSb, enables to design thicker barrier layers and hence relaxes the demands on growth accuracy. Best devices work up to $142 \mathrm{~K}$. Moreover, broadband lasing over a range of $660 \mathrm{GHz}$ is observed, making the devices already potential candidates for tuning and mode-locking experiments. The interface asymmetry in InGaAs/GaAsSb and the unipolar transport in QCLs allow for highly interesting studies. Nominally symmetric structures exhibit a significant bias polarity dependence of the transport and lasing performance. The results show that InGaAs/GaAsSb $\mathrm{THz}$ QCLs have to operated/grown in a certain direction in order to obtain the best performance [6]. This demonstrates that the growth direction can have a significant influence on QCLs and is a direct experimental proof of the importance of interface roughness scattering. Considering such growth-induced imperfections, optimized band structures, adapted doping profiles and interface engineering create a lot of room for improvements. InGaAs based $\mathrm{THz}$ QCLs definitely have the potential to outperform GaAs/AlGAs THz QCLs.

A further reduction of the effective mass is possible by using InAs wells and AlAsSb barriers. Due to the very high conduction band offset only very thin barriers can be employed which makes the design and growth very demanding. We have fabricated an InAs/AlAsSb QCL structure which shows lasing at $3.8 \mathrm{THz}$ in a magnetic field [8]

\section{ACKNOWLEDGMENT}

We acknowledge financial support by the Austrian Science Foundation through SFB NextLite (F49), IR-ON (F25) and the Doctoral School DK Solids for Function

\section{REFERENCES}

[1] M. Krall et al.,"Subwavelength micropillar array terahertz lasers", Optics Express 22, 274 (2014); doi:10.1364/OE.22.000274

[2] M. Fischer et al., Appl. Phys. Lett. 97, 221114 (2010).

[3] M. Nobile et al., Electronics Letters 45, 1031 (2009).

[4] S. Kumar, IEEE J. Quantum Electron. 17, 38 (2011).

[5] C. Deutsch et al., "Probing scattering mechanisms with symmetric quantum cascade lasers", Optics Express 21, 7209 (2013); doi: 10.1364/OE.21.007209

[6] C. Deutsch et al., "High performance InGaAs/GaAsSb terahertz quantum cascade lasers operating up to $142 \mathrm{~K}$ "; Applied Physics Letters, 101 (2012), 21; S. 2111171 - 2111174.

[7] C. Deutsch et al., "InGaAs/GaAsSb terahertz quantum cascade lasers", Journal of Infrared, Millimeter and Terahertz Waves 34, 374-385 (2013); doi: 10.1007/s10762-013-9991-5

[8] M. Brandstetter et al., "InAs based terahertz quantum cascade lasers", Appl. Phys. Lett. 108, $011109 \quad$ (2016); http://dx.doi.org/10.1063/1.4939551

(c) (c) 2016 IEEE. Personal use of this material is permitted. Permission from IEEE must be obtained for all other uses, in any current or future media, including reprinting/republishing this material for advertising or promotional purposes, creating new collective works, for resale or redistribution to servers or lists, or reuse of any copyrighted component of this work in other works.

This is the accepted version of M. Brandstetter et al., "THz Quantum Cascade Lasers with low effective mass active region," 2016 IEEE Photonics Conference (IPC), Waikoloa, USA, 2016, doi: 10.1109/IPCon.2016.7830983 